\title{
Evolution, trends and design parameters for the management of Areas of Logistic Activities in Spain
}

\section{Evolución, tendencias y parámetros de diseño para la gestión de Áreas de Actividades Logísticas en España}

José Romero Postiguillo (Main and Corresponding Author)

Comunidad de Madrid

CL. de Modesto Lafuente no 5, 6ㅇ, 4. 28010, Madrid (Spain)

joseromero@toledo.es

José María Del Campo Yagüe

Universidad Politécnica de Madrid

CL. Alfonso XII, 3 y 5. 28014, Madrid (Spain)

josemaria.delcampo@upm.es

Juan Antonio Santamera Sánchez

Universidad Politécnica de Madrid

Av. Ramiro de Maeztu s/n. 28040, Madrid (Spain)

juanantonio.santamera@upm.es

Manuscript Code: 708

Date of Acceptance/Reception: 24.07.2017/14.11.2015

DOI: 10.7764/RDLC.16.2.274

\begin{abstract}
In the last 50 years a new organization of international markets that directly affects procurement systems, production, and distribution has been developed, the supply chain. In this new globalized trade setup logistics is particularly relevant and unique, occupying preferential status in the competitiveness of products and companies. The transport system necessary for the supply and distribution is linked to linear and nodal infrastructures, both essential for the logistic chain. Within the nodal infrastructure, we find Logistics Platforms, which not only act as support to the infrastructure itself, but also provide value-added services and are configured as the main points of the supply chain, providing companies with the ability to implement some of the above mentioned logistic activities, in order to match demand and supply, thus optimizing the supply chain and reducing logistics' costs. Since there is no regulation and standardization to support the design of these "Logistics Areas", we will proceed to analyze 33 emblematic Spanish Logistics Platforms. This study will focus on the analysis of the role or relevance of logistics centrality, its intermodality, the degree of spatial concentration, the multifunctional level or the sector-specific expertise, and internal management. As a final point, the paper offers recommendations and standards for its design and management as well as a new definition of Logistics Activity Area.
\end{abstract}

Key words: LAA, Logistic Activity Area, logistic platform, urbanistic parameters, Spain.

Resumen

En los últimos 50 años se ha desarrollado una nueva organización de los mercados internacionales que afecta de forma directa a los sistemas de aprovisionamiento, producción y distribución, esto es, a la cadena de suministro. En esta nueva configuración globalizada del comercio adquiere una especial relevancia y singularidad la logística, posicionándose en el escalón preferente en la competitividad de productos y empresas. El sistema de transporte necesario para ese aprovisionamiento y distribución se encuentra ligado a infraestructuras lineales e infraestructuras nodales, ambas imprescindibles para la cadena logística. Dentro de las infraestructuras nodales nos encontramos las Plataformas Logísticas, las cuales no actúan únicamente como apoyo a la propia infraestructura sino que proporcionan servicios de valor añadido y se configuran como puntos básicos de la cadena de suministro. Dado que no se dispone de una reglamentación que normalice el diseño de estos "Espacios Logísticos" en relación con el transporte terrestre, se ha procedido al análisis de 33 Plataformas Logísticas españolas representativas (40\% muestral de las que están en funcionamiento en la actualidad en el territorio nacional) sobre las que se ha centrado el estudio sobre cada una de ellas en el análisis del rol o grado de centralidad logística, la intermodalidad, el grado de concentración espacial, el nivel de multifuncionalidad o especialización sectorial, los servicios ofertados, y la ordenación interna. Finalmente, el artículo concluye con unas recomendaciones y estándares para su diseño y ordenación así como con una nueva definición de "Zona de Actividad Logística".

Palabras clave: ZAL, Área de Actividad Logística, plataforma logística, parámetros urbanísticos, España.

Introduction

Since its creation in 1957, the EEC (the Treaty establishing the European Economic Community) has been provided with a common policy in the transport sector whose main goal was, among others, abolishing, thus, customs duties and quantitative restrictions on incoming and outgoing freight between Member States and establishing a Common Customs Tariff and a common trade policy in third countries. This was the first step to determine a posteriori the 
transport policies of the European Union as well as the interrelationship between the various transport systems and, finally, the existence and need of the Logistic Activity Areas.

Therefore, common European policy has been reflected in the following White Papers on Transport:

- The Future Development of the Common Transport Policy. (Opening-up of the transport market) (Comisión Europea, 1992).

- European Transport Policy for 2010: Time to Decide. (Improvement of the transport sector by rebalancing and strengthening intermodality by way of the Marco Polo Programme) (Comisión Europea, 2001)

- Roadmap to a Single European Transport Area - Towards a competitive and resource efficient transport system. (The creation of competitive and sustainable transport that supports mobility by optimising the performance of multimodal logistic chains to form a Single European Transport Area) (Unión Europea, 2011)

As for the Spanish legislation, the first regulatory background regarding Logistics Platforms was developed in 1979 within the Plan Nacional de Centros de Transporte de Mercancías [National Plan for Freight Transport Centres] (Ragàs Prat, 2012)

In 1987, although the Ley de Ordenación del Transporte Terrestre [Spanish Law for Road Transport] (Boletín Oficial del Estado, 2013) does not plan the location of the Goods Transport Centres, it gathers a series of determining factors to be considered.

Subsequently, the Plan Estratégico de Infraestructuras y Transporte - PEIT [Strategic Infrastructure and Transport Plan] (Ministerio de Fomento, 2005) focuses on, among other things, the need for a Plan Intermodal de Mercancías [Intermodal Freight Transportation Plan] to develop an integrated transport system within a complementary and coordinated framework between the different methods and also between infrastructures and competition services of various Administrations and Institutions, and for the development of a network of regional intermodal platforms, embedded in the main areas of production and consumption at regional level.

Finally the Plan de Infraestructuras, Transporte y Vivienda - PITVI [Plan for Infraestructure, Transport and Housing] (Ministerio de Fomento, 2012) orientates the transport politic to a planning of response to the actual needs of modality, establishing the needs of the user and the customer at the center of the initiatives, liberalizing markets to generate increased competitiveness.

To this national regulation it should be added the ones corresponding to each of the Autonomous Regions (the only ones who have competence in this field), although they are mainly focused on the development of strategic Infrastructure Plans that, generally, plan out the geographical location of the various goods, the Integrated Centres or Transport Centres.

\section{Description of the problem}

Therefore, despite the intentions of the European Union to intervene in this regard, since the first appearance in France of what we now identify as the first worldwide Logistics Platform, GARONOR in 1967, and back to the first Centro de Transporte de Mercancías [Freight Transport Centre] recognized as such in Spain, the CTM Madrid in 1991, up to now, there is no specific, clear and standarized regulation that allows planning and designing homogeneously any Logistics Area.

This research paper aims at determining the common standards of general design parameters that would regulate later studies on implementation and development regarding Logistics Areas, which, during the first steps of the design stage, may ensure its functionality and meet the needs required once put into service for a specific year.

Similarly, it is intended to standardize the concept of "Logistics Space" (integral centre of goods, goods transport centre, logistics platform, logistics-industrial platform, etc.), Therefore, its purpose and functionality is clear.

Generally speaking, the most international common term when referring to different types of Logistics Platforms is Logistics Centre. 
By analysing documents on logistics centres, we find obvious that there is no agreement for typological classification or agreement on designation and functions of each type (Meidute, 2005; Notteboom \& Rodrigue, 2009; Rimiene \& Grundey, 2007; Rodrigue, Debrie, \& Fremont, 2010).

Terms have been vaguely defined, describing these centres as freight hub, Gateway freight, inland port, inland terminal, dry port and freight villages. These definitions cover a wide variety of roles and levels: from terminals with simple and specific functions to facilities where complex relations take place, including logistics areas and common management structures (Rodrigue et al., 2010).

As a result, the concept and definitions of these centres may lead us to confusion.

There are also divergences between countries as similar facilities are called differently. Tsamboulas \& Dimitropoulos (1999) call nodal centre of goods is referred what is known as freight villages in the United Kingdom, multi-modal platforms or logistiques in France, interporty in Italy, integrated freight centres in Spain, and gueterverkeherszentren (GVZ) in Germany.

The Spanish situation remains similar to international: there is no uniform approach, disparity of concepts and lack of uniformity criteria. Thus, we can find: Freight Integral Centre, Integrated Freight Centre, Goods Transport Centre, Transport Centre, Integrated Transport Centre, Transport City, Goods Integrated Head Office, Logistics Platform, Logistics Area, Logistics Park, Logistic-Industrial Platform, and Transport and Logistics Intermodal Centre.

In this sense, we conclude that the classification made by Higgins, Ferguson, \& Kanaroglou (2012) is the best fit to the international realities because they develop a unified type of hierarchy and typology of logistics centres using the defining variants of other authors. The result of this work is a hierarchy of intermodal logistics centres according to infrastructure size, the influence and role of goods in the regional area and logistics as well as value-added activities, which is reflected in the Table 1.

Table 1. Classification by Higgins, Ferguson \& Kanaroglou (2012).

\begin{tabular}{|c|c|c|}
\hline Classification & Description & Typology \\
\hline $\begin{array}{l}3^{\mathrm{RD}} \text { Level } \\
\text { Gateway Cluster }\end{array}$ & $\begin{array}{l}\text { The 3rd level defines the largest scope } \\
\text { of activities contained within major } \\
\text { international Mainport terminals. }\end{array}$ & $\begin{array}{l}\text { Mainport Terminal as the primary interface between maritime } \\
\text { and inland freight and logistics, mainport terminals act as nodal } \\
\text { centres for logistics that produce large amounts of activity both } \\
\text { inside the facility and within its periphery. }\end{array}$ \\
\hline $\begin{array}{l}2^{\mathrm{ND}} \text { Level } \\
\text { Freight Transportation } \\
\& \text { Distribution Cluster }\end{array}$ & $\begin{array}{l}\text { The activities performed by these } \\
\text { facilities range from the simple } \\
\text { transfer of goods from one mode to } \\
\text { anther at an intermodal terminal, to } \\
\text { the broad range of intermodal } \\
\text { transportation options, wide } \\
\text { geographic influence, and } \\
\text { comprehensive value added services } \\
\text { offered by freight villages. }\end{array}$ & $\begin{array}{l}\text { Intermodal Terminal is a facility dedicated to the transshipment } \\
\text { and consolidation of intermodal freight into bigger flows for } \\
\text { regional and continental trade. } \\
\text { Inland Port can be understood as an inland extension of a } \\
\text { traditional seaport, connected to the mainport terminal by a } \\
\text { high-capacity rail shuttle or barge link for short sea shipping. } \\
\text { Freight Village is a site or area hosting a cluster of industrial, } \\
\text { intermodal, distribution, and logistics infrastructure and } \\
\text { supporting services dedicated to facilitating the flow of goods. }\end{array}$ \\
\hline \multirow[t]{2}{*}{$\begin{array}{l}1^{\text {ST }} \text { Level } \\
\text { Warehousing \& } \\
\text { Distribution Cluster }\end{array}$} & $\begin{array}{l}\text { The 1st level represents the smallest } \\
\text { scope of activities performed by } \\
\text { intermodal logistics centres. }\end{array}$ & $\begin{array}{l}\text { Warehouses are typically a place for inventory and storage and } \\
\text { perform the basic function of acting as a buffer between } \\
\text { suppliers, manufacturers, and customers to smooth time and } \\
\text { demand constraints in the supply chain. }\end{array}$ \\
\hline & $\begin{array}{l}\text { Warehousing and Distribution Centres } \\
\text { perform a variety of logistics functions } \\
\text { and serve as important basic elements } \\
\text { in the supply chain. }\end{array}$ & $\begin{array}{l}\text { Distribution Centre are a single large warehouse or cluster of } \\
\text { warehouses dedicated to the rapid movement of goods. } \\
\text { Container Yard is a facility dedicated to performing the basic } \\
\text { functions of storage, cleaning, and repair of empty containers. } \\
\text { Inland Container Depot offer a location for the handling and } \\
\text { temporary storage of containerized trade. }\end{array}$ \\
\hline
\end{tabular}

In Spain the most widely accepted definitions are the Integrated Goods Centre "a set of facilities and equipment where various activities directly related to national or international transport take place, which can be used together as a logistics centre for manufacturers and retailers due to their relationship with the transport sector and have a range of complementary services available to different users (Haulage contractor, industrials, warehouse owner, dealers, agents, etc.)" (Coca, Colomer \& Aznar, 2010) and the Transportation Centre "(also known as Centre for Transportation and Logistics, Logistics Centre, Logistics Platform, Freight Platform or Goods Station Area in which we find all the activities related to transport (e), logistics and distribution of goods (a) for both domestic and international 
traffic, may intervene several companies in the Transport sector" ("Asociación de Centros de Transporte de España," n.d.) and Europlatforms ("The European Logistics Platforms Association," n.d.).

The methodology used to carry out this research was initially based on the study of the evolution of the Logistics Platform's concept. To this aim, we have analyzed the evolution of the concept and determined the relationship between the existing definitions, as they show different functions.

As for the analysis of Logistics Platforms in Spain, we have classified them according to their geographical location and degree of logistics' centrality (intermodal, level of spatial concentration, sector-specific expertise, and its internal organization), the design urban parameters (minimum size plot, plot's front, plot's maximum capacity, building height. and building's number of floors), and the various services offered in all Logistics Platforms (number of service stations providing petroleum fuels products, number of service stations providing natural gas, MOT Facilities, day-care centre services, number of bank branches, sports area, post office/postal services, availability of training classrooms, availability of meeting romos, driving school services, number of restaurants and/or cafés, public transport connection to town centre, vehicle rental services, customs services and number of hotels).

For data gathering purposes we used 33 logistics platforms, which represent around $40 \%$ of those currently operational within Spain. The table 2 shows the parameters of Surface, role or centrality, concentration and level of functionality of the Logistics platforms review.

\begin{tabular}{|c|c|c|c|c|c|c|}
\hline Logistic platform & $\begin{array}{c}\text { Surface } \\
\left(\mathrm{m}^{2}\right)\end{array}$ & $\begin{array}{c}\text { Role or } \\
\text { Centrality }\end{array}$ & Intermodal & Modes & Concentration & $\begin{array}{c}\text { Level of } \\
\text { Functionality }\end{array}$ \\
\hline Centro de Transporte de Madrid (CTM) & 338,500 & International & Monomodal & 1 & Single-centre & Multifunctional \\
\hline Plataforma Logística de Zaragoza (PLAZA) & $12,826,898$ & International & Multimodal & 3 & Single-centre & Multifunctional \\
\hline Ciudad del Transporte de Pamplona & $1,622,587$ & International & Multimodal & 2 & Single-centre & Multifunctional \\
\hline Centro de Transportes de Coslada & $1,078,865$ & International & Monomodal & 1 & Single-centre & Multifunctional \\
\hline Plataforma logística El Sequero & $1,513,657$ & Domestic & Monomodal & 1 & Single-centre & Multifunctional \\
\hline Centro Integrado Transporte de Murcia & 696,591 & Domestic & Monomodal & 1 & Single-centre & Multifunctional \\
\hline Central Integrada de Mercancías CIM EI Vallés & 696,591 & Domestic & Monomodal & 1 & Single-centre & Multifunctional \\
\hline Central Integrada de Mercancías CIM El Camp & 423,108 & Domestic & Monomodal & 1 & Single-centre & Multifunctional \\
\hline Miranda Logística & $2,496,861$ & Domestic & Monomodal & 1 & Single-centre & Multifunctional \\
\hline Centro de Ttes. de Mercancías de Córdoba. & 368,106 & Domestic & Monomodal & 1 & Single-centre & Multifunctional \\
\hline Parque logístico-empresarial La Alquería. & $1,839,430$ & Domestic & Monomodal & 1 & Single-centre & Multifunctional \\
\hline Plataforma Logística de Huesca (PLAHUS) & $1,157,062$ & Domestic & Multimodal & 2 & Single-centre & Multifunctional \\
\hline Plataforma Logística de Teruel (PLATEA) & $2,545,510$ & Domestic & Multimodal & 2 & Single-centre & Multifunctional \\
\hline Plataforma Logística de Fraga (PLAFRAGA) & 855,931 & Domestic & Monomodal & 1 & Single-centre & Multifunctional \\
\hline Centro de Transporte de Bailén. & 320,181 & Domestic & Monomodal & 1 & Single-centre & Multifunctional \\
\hline Centro de Ttes. de Bizcaia (APARKABISA) & 312,560 & International & Monomodal & 1 & Multisite & Multifunctional \\
\hline Parque Logístico ARASUR & $2,000,000$ & International & Monomodal & 1 & Single-centre & Multifunctional \\
\hline Centro Tte. Mercancías de Salamanca & 668,890 & International & Multimodal & 2 & Single-centre & Multifunctional \\
\hline Centro de Transportes de Benavente & 220,000 & International & Monomodal & 1 & Single-centre & Multifunctional \\
\hline Centro de Transportes Aduana de Burgos & $161,874.26$ & International & Multimodal & 2 & Single-centre & Multifunctional \\
\hline Centro de Transportes de Gijón & 150,000 & International & Monomodal & 1 & Single-centre & Multifunctional \\
\hline Centro de Transportes de Segovia & 200,000 & Domestic & Monomodal & 1 & Single-centre & Multifunctional \\
\hline CIM y Logística de Vitoria & 715,000 & International & Multimodal & 2 & Single-centre & Multifunctional \\
\hline Central Integrada de Mercancías El Ejido & 180,000 & Domestic & Monomodal & 1 & Single-centre & Multifunctional \\
\hline Centro Integrado Mercancías CIM Valladolid & $1,095,709$ & National & Monomodal & 1 & Single-centre & Multifunctional \\
\hline Centro de Transportes de León & 265,000 & National & Monomodal & 1 & Single-centre & Multifunctional \\
\hline Central Integrada de Mercancías CIM La Selva & $225,479.10$ & National & Multimodal & 2 & Single-centre & Multifunctional \\
\hline Central Integrada de Mercancías CIM de Lleida & 420,000 & National & Monomodal & 1 & Single-centre & Multifunctional \\
\hline Ciudad del Transporte de Santander & 100,000 & National & Monomodal & 1 & Single-centre & Multifunctional \\
\hline Ciudad del Transporte de Zaragoza & 605,000 & National & Monomodal & 1 & Single-centre & Multifunctional \\
\hline Centro Transporte Mercancías de Málaga & 226,325 & National & Monomodal & 1 & Single-centre & Multifunctional \\
\hline Centro Transporte Mercancías de Sevilla & 252,745 & National & Multimodal & 2 & Single-centre & Multifunctional \\
\hline Plataforma Logística de Irún (ZAISA) & 400,000 & International & Monomodal & 1 & Multisite & Multifunctional \\
\hline
\end{tabular}


Forty percent of the platforms analysed show a degree of international centrality while the remaining $60 \%$ function on a national/regional level.

Likewise, as far as intermodality is concerned, $70 \%$ of them are not intermodal, in other words they are exclusively road-transport based, and practically all of them present a degree of "single-centre" concentration.

Surface area distribution is uniform. Having said this, with the exception of the Zaragoza PLAZA logistics platform Figure 1 shows that they can be grouped into three intervals consisting of platforms of up to 50 ha, of between 50 and 100 ha, and of between 100 and 200 ha. The three special cases of Miranda Logística (250 ha), PLATEA (250 ha) and PLAZA (1,280 ha) are not included in this breakdown.

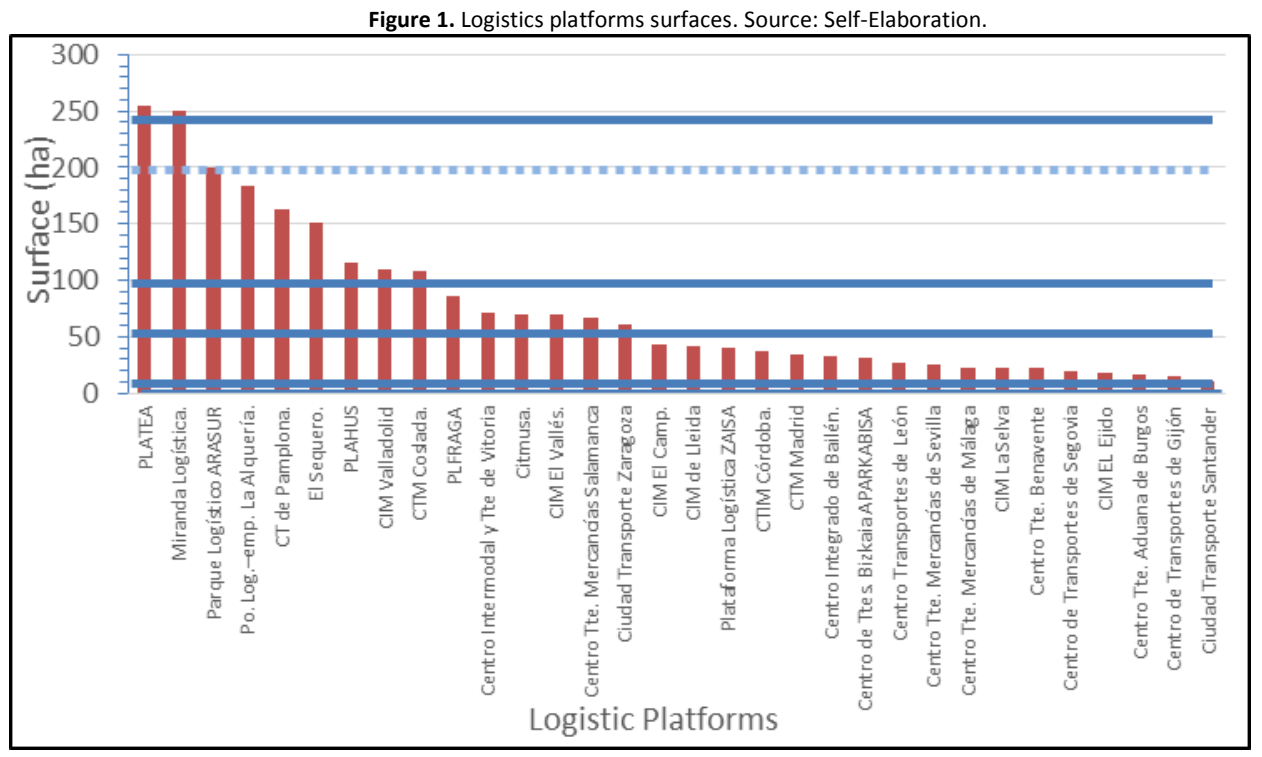

With respect to services there is no relation between the number of services offered, the nature thereof and the surface of the logistics platform. However, mention must be made of the fact that basic services such as restaurants/cafeterias and petrol stations which might attract external customers to the platform are provided at more than $80 \%$ of them.

A similar situation can be found at $50 \%$ of the platforms with respect to hotel and public transport services.

Table 3 lists the different services offered by the logistics platforms and the presence percentage thereof.

\begin{tabular}{lc} 
Table 3. Presence of services offered at the logistics platforms. \\
\hline Service offered & \% Logistics platforms \\
\hline Restaurants / Cafes & 87 \\
Petrol station & 83 \\
Meeting rooms & 52 \\
Hotel & 48 \\
Public Transport & 48 \\
Training classrooms & 43 \\
Customs services & 43 \\
Bank branches & 39 \\
MOT facilities & 26 \\
Post office / Postal services & 26 \\
Vehicle rental services & 17 \\
Driving school services & 13 \\
Natural gas station & 9 \\
Day-care centre services & 9 \\
Sport area & 4 \\
Shopping area & 4 \\
\hline Source: Self-Elaboration. &
\end{tabular}


As far as urban design parameters are concerned, the following tables (4 to 6 ) show the values with respect to the surfaces of each functional area, the building coefficients thereof and the specific plot planning parameters for each functional area. These parameters were obtained from the different municipal planning documents on which each of the logistics platforms analysed are based.

Table 4. Building ratio.

\begin{tabular}{|c|c|c|c|}
\hline $\begin{array}{l}\text { Minimum Coefficient } \\
\text { Building }\left(\mathrm{m}^{2} / \mathrm{m}^{2}\right)\end{array}$ & $\begin{array}{l}\text { Maximum Coefficient } \\
\text { Building }\left(\mathrm{m}^{2} / \mathrm{m}^{2}\right)\end{array}$ & $\begin{array}{l}\text { Average Coefficient } \\
\text { Building }\left(\mathrm{m}^{2} / \mathrm{m}^{2}\right)\end{array}$ & $\begin{array}{l}\text { Weighted Coefficient } \\
\text { Building }\left(\mathrm{m}^{2} / \mathrm{m}^{2}\right)\end{array}$ \\
\hline \multicolumn{4}{|l|}{ Logistic platform } \\
\hline 0.332 & 0.595 & 0.476 & 0.495 \\
\hline \multicolumn{4}{|l|}{ Logistic area } \\
\hline 0.311 & 1.2 & 0.822 & 0.970 \\
\hline \multicolumn{4}{|l|}{ Vehicle service area } \\
\hline 0.2 & 2.6 & 0.953 & 1.099 \\
\hline \multicolumn{4}{|c|}{ Service and administration centre } \\
\hline 0.6 & 2.6 & 1.142 & 1.075 \\
\hline \multicolumn{4}{|l|}{ Intermodal facilities } \\
\hline 0.33 & 0.64 & 0.478 & 0.615 \\
\hline
\end{tabular}

\begin{tabular}{|c|c|c|}
\hline \multicolumn{3}{|c|}{ Logistic Area } \\
\hline $\begin{array}{l}\text { Minimum } \\
\text { surface } \\
\left(\mathrm{m}^{2}\right)\end{array}$ & $\begin{array}{c}\text { MAXIMUM } \\
\text { Surface } \\
\left(\mathrm{m}^{2}\right)\end{array}$ & $\begin{array}{c}\text { Average } \\
\text { Surface }\left(\mathrm{m}^{2}\right)\end{array}$ \\
\hline 70,904 & $3,233,828$ & $505,736.071$ \\
\hline $\begin{array}{c}\text { Minimum } \\
\text { percentage }\end{array}$ & $\begin{array}{l}\text { MAXIMUM } \\
\text { percentage }\end{array}$ & $\begin{array}{c}\text { Average } \\
\text { percentage }\end{array}$ \\
\hline 4.68 & 48.98 & 33.51 \\
\hline \multicolumn{3}{|c|}{ Vehicle service area } \\
\hline $\begin{array}{c}\text { Minimum } \\
\text { surface } \\
\left(\mathrm{m}^{2}\right)\end{array}$ & $\begin{array}{c}\text { MAXIMUM } \\
\text { Surface } \\
\left(\mathrm{m}^{2}\right)\end{array}$ & $\begin{array}{c}\text { Average } \\
\text { Surface }\left(\mathrm{m}^{2}\right)\end{array}$ \\
\hline $4,435.41$ & 101,891 & $25,657.786$ \\
\hline $\begin{array}{l}\text { Minimum } \\
\text { percentage }\end{array}$ & $\begin{array}{l}\text { MAXIMUM } \\
\text { percentage }\end{array}$ & $\begin{array}{c}\text { Average } \\
\text { percentage }\end{array}$ \\
\hline 0.38 & 6.09 & 2.5 \\
\hline \multicolumn{3}{|c|}{ Truck parking space } \\
\hline $\begin{array}{c}\text { Minimum } \\
\text { surface } \\
\left(\mathrm{m}^{2}\right)\end{array}$ & $\begin{array}{c}\text { MAXIMUM } \\
\text { Surface } \\
\left(\mathrm{m}^{2}\right)\end{array}$ & $\begin{array}{c}\text { Average } \\
\text { Surface }\left(\mathrm{m}^{2}\right)\end{array}$ \\
\hline 6,511 & 170,273 & 45,681 \\
\hline $\begin{array}{l}\text { Minimum } \\
\text { percentage }\end{array}$ & $\begin{array}{l}\text { MAXIMUM } \\
\text { percentage }\end{array}$ & $\begin{array}{c}\text { Average } \\
\text { percentage }\end{array}$ \\
\hline 0.97 & 13.51 & 4.421 \\
\hline
\end{tabular}

\begin{tabular}{ccc}
\multicolumn{3}{c}{ Table 5. Platforms surfaces and funtional areas. } \\
\hline \multicolumn{3}{c}{ Logistics platforms review } \\
\hline $\begin{array}{c}\text { Minimum } \\
\text { surface }\left(\mathrm{m}^{2}\right)\end{array}$ & $\begin{array}{c}\text { Maximum } \\
\text { Surface } \\
\left(\mathrm{m}^{2}\right)\end{array}$ & $\begin{array}{c}\text { Average } \\
\text { Surface } \\
\left(\mathrm{m}^{2}\right)\end{array}$ \\
\hline 320,181 & $12,826,898$ & $\begin{array}{c}1,932,338.0 \\
6\end{array}$ \\
\hline
\end{tabular}

\begin{tabular}{|c|c|c|c|c|c|}
\hline \multicolumn{3}{|c|}{ Service and Administration centre } & \multicolumn{3}{|c|}{ Intermodal facilities } \\
\hline & MAXIMUM & Average & \multicolumn{3}{|c|}{ MAXIMUM } \\
\hline $\begin{array}{c}\text { Minimum } \\
\text { surface }\left(m^{2}\right)\end{array}$ & $\begin{array}{c}\text { Surface } \\
\left(\mathrm{m}^{2}\right)\end{array}$ & $\begin{array}{l}\text { Surface } \\
\left(\mathrm{m}^{2}\right)\end{array}$ & $\begin{array}{c}\text { Minimum } \\
\text { surface }\left(\mathrm{m}^{2}\right)\end{array}$ & $\begin{array}{l}\text { Surface } \\
\left(\mathrm{m}^{2}\right)\end{array}$ & $\begin{array}{c}\text { Average } \\
\text { Surface }\left(\mathrm{m}^{2}\right)\end{array}$ \\
\hline $5,546.09$ & $1,552,804$ & 143,541 & 122,251 & $2,933,411$ & $1,041,894.75$ \\
\hline $\begin{array}{l}\text { Minimum } \\
\text { percentage }\end{array}$ & $\begin{array}{l}\text { MAXIMUM } \\
\text { percentage }\end{array}$ & $\begin{array}{c}\text { Average } \\
\text { percentage }\end{array}$ & $\begin{array}{l}\text { Minimum } \\
\text { percentage }\end{array}$ & $\begin{array}{l}\text { MAXIMUM } \\
\text { percentage }\end{array}$ & $\begin{array}{c}\text { Average } \\
\text { percentage }\end{array}$ \\
\hline 0.48 & 17.02 & 5.11 & 7.53 & 35.88 & 23.415 \\
\hline \multicolumn{3}{|c|}{ Road network } & \multicolumn{3}{|c|}{ Industrial area } \\
\hline & MAXIMUM & Average & \multicolumn{3}{|c|}{ MAXIMUM } \\
\hline $\begin{array}{c}\text { Minimum } \\
\text { surface }\left(\mathrm{m}^{2}\right)\end{array}$ & $\begin{array}{c}\text { Surface } \\
\left(\mathrm{m}^{2}\right)\end{array}$ & $\begin{array}{c}\text { Surface } \\
\left(\mathrm{m}^{2}\right)\end{array}$ & $\begin{array}{c}\text { Minimum } \\
\text { surface }\left(\mathrm{m}^{2}\right)\end{array}$ & $\begin{array}{c}\text { Surface } \\
\left(\mathrm{m}^{2}\right)\end{array}$ & $\begin{array}{c}\text { Average } \\
\text { Surface }\left(\mathrm{m}^{2}\right)\end{array}$ \\
\hline 34,977 & $1,736,072$ & 296,026 & 13,614 & 623,276 & $361,511.25$ \\
\hline $\begin{array}{l}\text { Minimum } \\
\text { percentage }\end{array}$ & $\begin{array}{l}\text { MAXIMUM } \\
\text { percentage }\end{array}$ & $\begin{array}{c}\text { Average } \\
\text { percentage }\end{array}$ & $\begin{array}{l}\text { Minimum } \\
\text { percentage }\end{array}$ & $\begin{array}{l}\text { MAXIMUM } \\
\text { percentage }\end{array}$ & $\begin{array}{c}\text { Average } \\
\text { percentage }\end{array}$ \\
\hline 8.27 & 27.58 & 17.30 & 1.51 & 41.18 & 19.585 \\
\hline \multicolumn{3}{|c|}{ Green areas } & \multicolumn{3}{|c|}{ Area science and Technology } \\
\hline & MAXIMUM & Average & \multicolumn{3}{|c|}{ MAXIMUM } \\
\hline $\begin{array}{c}\text { Minimum } \\
\text { surface }\left(\mathrm{m}^{2}\right)\end{array}$ & $\begin{array}{c}\text { Surface } \\
\left(\mathrm{m}^{2}\right)\end{array}$ & $\begin{array}{c}\text { Surface } \\
\left(\mathrm{m}^{2}\right)\end{array}$ & $\begin{array}{c}\text { Minimum } \\
\text { surface }\left(\mathrm{m}^{2}\right)\end{array}$ & $\begin{array}{c}\text { Surface } \\
\left(\mathrm{m}^{2}\right)\end{array}$ & $\begin{array}{c}\text { Average } \\
\text { Surface }\left(\mathrm{m}^{2}\right)\end{array}$ \\
\hline $46,527.82$ & $2,192,987$ & 314,842 & $146,261.7$ & 203,604 & 175,298 \\
\hline $\begin{array}{l}\text { Minimum } \\
\text { percentage }\end{array}$ & $\begin{array}{l}\text { MAXIMUM } \\
\text { percentage }\end{array}$ & $\begin{array}{c}\text { Average } \\
\text { percentage }\end{array}$ & $\begin{array}{l}\text { Minimum } \\
\text { percentage }\end{array}$ & $\begin{array}{l}\text { MAXIMUM } \\
\text { percentage }\end{array}$ & $\begin{array}{c}\text { Average } \\
\text { percentage }\end{array}$ \\
\hline 9.98 & 23.86 & 16.03 & 5.86 & 11.63 & 8.50 \\
\hline
\end{tabular}


Table 6. Conditions of plot and construction in functional areas of a logistic platform.

\begin{tabular}{|c|c|c|c|}
\hline \multicolumn{4}{|c|}{ Logistic area } \\
\hline Minimum Plot & $\begin{array}{l}\text { MAXIMUM } \\
\text { Plot }\end{array}$ & $\begin{array}{l}\text { Average } \\
\text { Plot }\end{array}$ & $\begin{array}{c}\text { Weighted } \\
\text { Average Plot }\end{array}$ \\
\hline $200 \mathrm{~m}^{2}$ & $10,000 \mathrm{~m}^{2}$ & $3,290 \mathrm{~m}^{2}$ & $4,165.608 \mathrm{~m}^{2}$ \\
\hline $\begin{array}{c}\text { Minimum Plot's } \\
\text { Front }\end{array}$ & $\begin{array}{l}\text { MAXIMUN } \\
\text { Plot's Front }\end{array}$ & $\begin{array}{c}\text { Average } \\
\text { Plot's Front }\end{array}$ & $\begin{array}{c}\text { Weighted } \\
\text { Average Plot's } \\
\text { Front }\end{array}$ \\
\hline $0 \mathrm{~m}$ & $50 \mathrm{~m}$ & $27.111 \mathrm{~m}$ & $28.133 \mathrm{~m}$ \\
\hline $\begin{array}{c}\text { Plot's } \\
\text { Maximum } \\
\text { capacity } \\
\text { minimum } \\
\end{array}$ & $\begin{array}{c}\text { Plot's } \\
\text { Maximum } \\
\text { capacity } \\
\text { MAXIMUM }\end{array}$ & $\begin{array}{l}\text { Average } \\
\text { Plot's } \\
\text { Maximum } \\
\text { capacity } \\
\end{array}$ & $\begin{array}{l}\text { Weighted } \\
\text { Average Plot's } \\
\text { Maximum } \\
\text { capacity }\end{array}$ \\
\hline $40 \%$ & $100 \%$ & $73.472 \%$ & $72.597 \%$ \\
\hline $\begin{array}{l}\text { Building height } \\
\text { Maximum } \\
\text { minimum }\end{array}$ & $\begin{array}{l}\text { Building } \\
\text { height } \\
\text { Maximum } \\
\text { MAXIMUM }\end{array}$ & $\begin{array}{c}\text { Average } \\
\text { Building } \\
\text { height } \\
\text { Maximum } \\
\end{array}$ & $\begin{array}{c}\text { Weighted } \\
\text { Average } \\
\text { Building height }\end{array}$ \\
\hline $7 \mathrm{~m}$ & $22 \mathrm{~m}$ & $14.571 \mathrm{~m}$ & $14.986 \mathrm{~m}$ \\
\hline $\begin{array}{c}\text { Building's } \\
\text { Minimum num. } \\
\text { of floors }\end{array}$ & $\begin{array}{c}\text { Building's } \\
\text { MAXIMUM } \\
\text { num. of floors }\end{array}$ & $\begin{array}{c}\text { Average } \\
\text { Building's } \\
\text { num. of floors }\end{array}$ & $\begin{array}{c}\text { Weighted } \\
\text { Average } \\
\text { Building's num. } \\
\text { of floors }\end{array}$ \\
\hline 2 & 5 & 3.3 & 3.516 \\
\hline \multicolumn{4}{|c|}{ Service and Administration centre } \\
\hline Minimum Plot & $\begin{array}{l}\text { MAXIMUM } \\
\text { Plot }\end{array}$ & $\begin{array}{l}\text { Average } \\
\text { Plot }\end{array}$ & $\begin{array}{c}\text { Weighted } \\
\text { Average Plot }\end{array}$ \\
\hline $500 \mathrm{~m}^{2}$ & $12,967.3 \mathrm{~m}^{2}$ & $3,776.236 \mathrm{~m}^{2}$ & $3,322.651 \mathrm{~m}^{2}$ \\
\hline $\begin{array}{c}\text { Minimum Plot's } \\
\text { Front }\end{array}$ & $\begin{array}{l}\text { MAXIMUM } \\
\text { Plot's } \\
\text { Front }\end{array}$ & $\begin{array}{c}\text { Average } \\
\text { Plot's Front }\end{array}$ & $\begin{array}{c}\text { Weighted } \\
\text { Average Plot's } \\
\text { Front }\end{array}$ \\
\hline $10 \mathrm{~m}$ & $40 \mathrm{~m}$ & $25 \mathrm{~m}$ & $28.568 \mathrm{~m}$ \\
\hline $\begin{array}{c}\text { Plot's } \\
\text { Maximum } \\
\text { capacity } \\
\text { minimum } \\
\end{array}$ & $\begin{array}{c}\text { Plot's } \\
\text { Maximum } \\
\text { capacity } \\
\text { MAXIMUM }\end{array}$ & $\begin{array}{l}\text { Average } \\
\text { Plot's } \\
\text { Maximum } \\
\text { capacity } \\
\end{array}$ & $\begin{array}{c}\text { Weighted } \\
\text { Average Plot's } \\
\text { Maximum } \\
\text { capacity }\end{array}$ \\
\hline $40 \%$ & $100 \%$ & $56.498 \%$ & $62.522 \%$ \\
\hline $\begin{array}{l}\text { Building height } \\
\text { Maximum } \\
\text { minimum }\end{array}$ & $\begin{array}{l}\text { Building } \\
\text { height } \\
\text { Maximum } \\
\text { MAXIMUM }\end{array}$ & $\begin{array}{l}\text { Average } \\
\text { Building } \\
\text { height } \\
\text { Maximum } \\
\end{array}$ & $\begin{array}{c}\text { Weighted } \\
\text { Average } \\
\text { Building height }\end{array}$ \\
\hline $4 \mathrm{~m}$ & $22 \mathrm{~m}$ & $17.417 \mathrm{~m}$ & $16.519 \mathrm{~m}$ \\
\hline $\begin{array}{c}\text { Building's } \\
\text { Minimum num. } \\
\text { of floors }\end{array}$ & $\begin{array}{c}\text { Building's } \\
\text { MAXIMUM } \\
\text { num. of floors }\end{array}$ & $\begin{array}{c}\text { Average } \\
\text { Building's } \\
\text { num. of floors }\end{array}$ & $\begin{array}{c}\text { Weighted } \\
\text { Average } \\
\text { Building's num. } \\
\text { of floors } \\
\end{array}$ \\
\hline 2 & 12 & 5.393 & 4.541 \\
\hline
\end{tabular}

\begin{tabular}{|c|c|c|c|}
\hline \multicolumn{4}{|c|}{ Vehicle service area } \\
\hline $\begin{array}{l}\text { Minimum } \\
\text { Plot }\end{array}$ & $\begin{array}{l}\text { MAXIMUM } \\
\text { Plot }\end{array}$ & $\begin{array}{c}\text { Average } \\
\text { Plot }\end{array}$ & $\begin{array}{c}\text { Weighted } \\
\text { Average Plot }\end{array}$ \\
\hline $250 \mathrm{~m}^{2}$ & $10,000 \mathrm{~m}^{2}$ & $3,544.20 \mathrm{~m}^{2}$ & $4,125.144 \mathrm{~m}^{2}$ \\
\hline $\begin{array}{l}\text { Minimum } \\
\text { Plot's Front }\end{array}$ & $\begin{array}{l}\text { MAXIMUN } \\
\text { Plot's Front }\end{array}$ & $\begin{array}{c}\text { Average } \\
\text { Plot's Front }\end{array}$ & $\begin{array}{c}\text { Weighted } \\
\text { Average Plot's } \\
\text { Front }\end{array}$ \\
\hline $10 \mathrm{~m}$ & $35 \mathrm{~m}$ & $27.143 \mathrm{~m}$ & $28.427 \mathrm{~m}$ \\
\hline $\begin{array}{c}\text { Plot's } \\
\text { Maximum } \\
\text { capacity } \\
\text { minimum }\end{array}$ & $\begin{array}{c}\text { Plot's } \\
\text { Maximum } \\
\text { capacity } \\
\text { MAXIMUM }\end{array}$ & $\begin{array}{l}\text { Average } \\
\text { Plot's } \\
\text { Maximum } \\
\text { capacity }\end{array}$ & $\begin{array}{c}\text { Weighted } \\
\text { Average Plot's } \\
\text { Maximum } \\
\text { capacity }\end{array}$ \\
\hline $20 \%$ & $100 \%$ & $53.545 \%$ & $63.052 \%$ \\
\hline $\begin{array}{l}\text { Building } \\
\text { height } \\
\text { Maximum } \\
\text { minimum }\end{array}$ & $\begin{array}{c}\text { Building } \\
\text { height } \\
\text { Maximum } \\
\text { MAXIMUM }\end{array}$ & $\begin{array}{c}\text { Average } \\
\text { Building } \\
\text { height } \\
\text { Maximum }\end{array}$ & $\begin{array}{l}\text { Weighted } \\
\text { Average } \\
\text { Building height }\end{array}$ \\
\hline $7 \mathrm{~m}$ & $22 \mathrm{~m}$ & $13.751 \mathrm{~m}$ & $14.116 \mathrm{~m}$ \\
\hline $\begin{array}{l}\text { Building's } \\
\text { Minimum } \\
\text { num. of } \\
\text { floors }\end{array}$ & $\begin{array}{c}\text { Building's } \\
\text { MAXIMUM } \\
\text { num. of floors }\end{array}$ & $\begin{array}{c}\text { Average } \\
\text { Building's } \\
\text { num. of floors }\end{array}$ & $\begin{array}{c}\text { Weighted } \\
\text { Average } \\
\text { Building's } \\
\text { num. of floors }\end{array}$ \\
\hline 2 & 10 & 3.869 & 4.107 \\
\hline \multicolumn{4}{|c|}{ Intermodal facilities } \\
\hline $\begin{array}{c}\text { Minimum } \\
\text { Plot }\end{array}$ & $\begin{array}{l}\text { MAXIMUM } \\
\text { Plot }\end{array}$ & $\begin{array}{c}\text { Average } \\
\text { Plot }\end{array}$ & $\begin{array}{c}\text { Weighted } \\
\text { Average Plot }\end{array}$ \\
\hline $4,000 \mathrm{~m}^{2}$ & $30,000 \mathrm{~m}^{2}$ & $13,833.33 \mathrm{~m}^{2}$ & $8,248.953 \mathrm{~m}^{2}$ \\
\hline $\begin{array}{l}\text { Minimum } \\
\text { Plot's Front }\end{array}$ & $\begin{array}{l}\text { MAXIMUM } \\
\text { Plot's } \\
\text { Front }\end{array}$ & $\begin{array}{c}\text { Average } \\
\text { Plot's Front }\end{array}$ & $\begin{array}{c}\text { Weighted } \\
\text { Average Plot's } \\
\text { Front }\end{array}$ \\
\hline ------- m & ------- m & ------- m & -------- m \\
\hline $\begin{array}{c}\text { Plot's } \\
\text { Maximum } \\
\text { capacity } \\
\text { minimum }\end{array}$ & $\begin{array}{c}\text { Plot's } \\
\text { Maximum } \\
\text { capacity } \\
\text { MAXIMUM }\end{array}$ & $\begin{array}{l}\text { Average } \\
\text { Plot's } \\
\text { Maximum } \\
\text { capacity }\end{array}$ & $\begin{array}{c}\text { Weighted } \\
\text { Average Plot's } \\
\text { Maximum } \\
\text { capacity }\end{array}$ \\
\hline $60 \%$ & $75 \%$ & $65 \%$ & $71.64 \%$ \\
\hline $\begin{array}{l}\text { Building } \\
\text { height } \\
\text { Maximum } \\
\text { minimum }\end{array}$ & $\begin{array}{c}\text { Building } \\
\text { height } \\
\text { Maximum } \\
\text { MAXIMUM }\end{array}$ & $\begin{array}{l}\text { Average } \\
\text { Building } \\
\text { height } \\
\text { Maximum }\end{array}$ & $\begin{array}{l}\text { Weighted } \\
\text { Average } \\
\text { Building height }\end{array}$ \\
\hline $13 \mathrm{~m}$ & $16 \mathrm{~m}$ & $14.5 \mathrm{~m}$ & $15.362 \mathrm{~m}$ \\
\hline $\begin{array}{l}\text { Building's } \\
\text { Minimum } \\
\text { num. of } \\
\text { floors }\end{array}$ & $\begin{array}{c}\text { Building's } \\
\text { MAXIMUM } \\
\text { num. of floors }\end{array}$ & $\begin{array}{c}\text { Average } \\
\text { Building's } \\
\text { num. of floors }\end{array}$ & $\begin{array}{l}\text { Weighted } \\
\text { Average } \\
\text { Building's } \\
\text { num. of floors }\end{array}$ \\
\hline 3 & 4 & 3.25 & 3.707 \\
\hline
\end{tabular}

Source: Self-Elaboration.

Finally, as regards the surfaces set aside within the logistics platforms for each functional area, no clear correlation exists between the theoretical use for which they are intended (logistics), other uses to which they are put, and the functions they should perform (vehicle service area and driver accommodation). Figure 2 highlights this situation, namely the diverse uses to which the logistics areas are put.

It can therefore be seen that although around $40 \%$ of the overall surface is used for logistics purposes, areas exist in which this percentage is below $5 \%$. In such cases can the facility really be described as a "logistics platform? In our opinion the answer is no. The same is true for the remaining functional areas. 
Figure 2. Surface of different functional areas in a logistics platform. Source: Self-Elaboration.

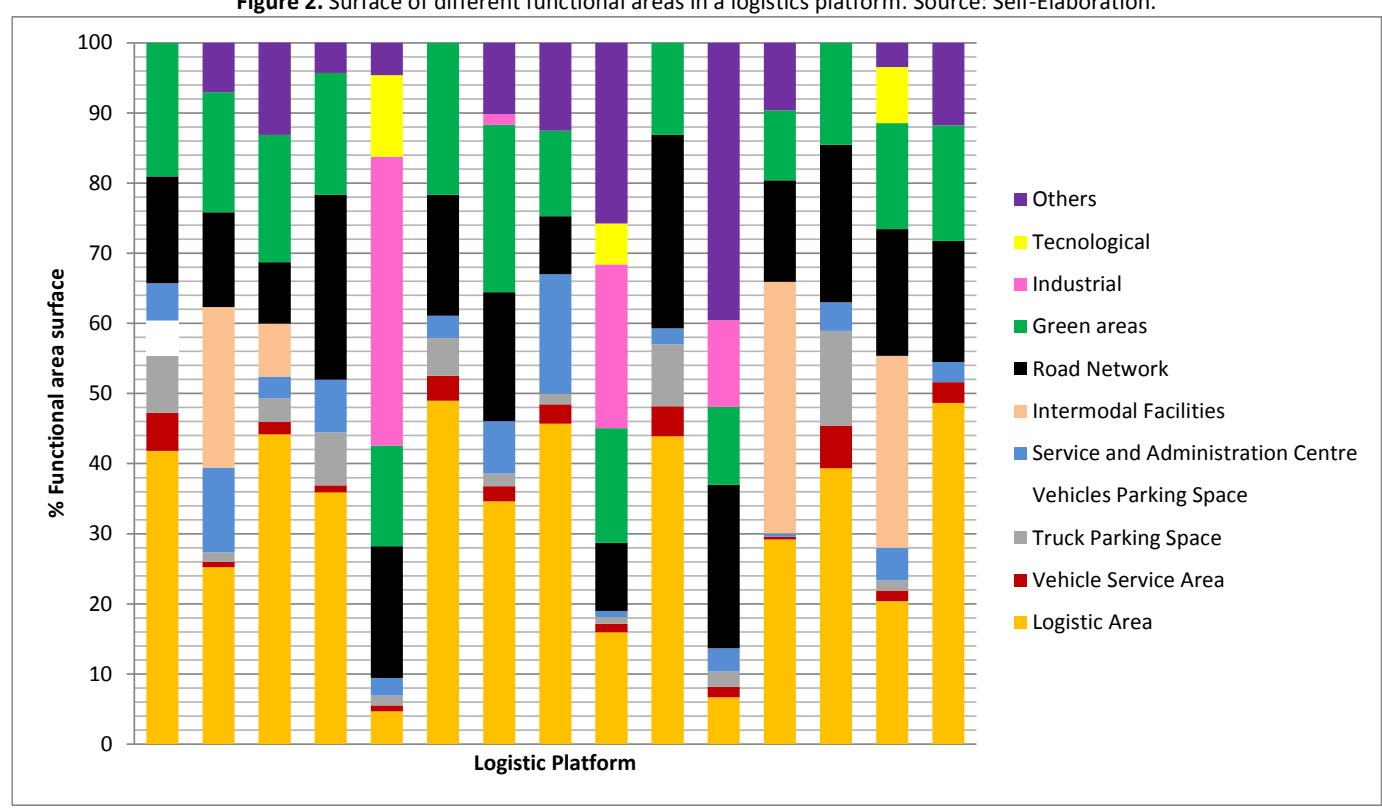

Conclusions

In light of the foregoing, our proposal is to homogenise the notion of the logistics platform to create a single concept we call the "LOGISTICS CENTRE" and which we define as a multifunctional nodal infrastructure for supporting the overland transport of freight, to which we shall add the appropriate adjectives that classify it in a clear and unmistakable manner:

- In accordance with its degree of centrality (international / national / regional / local), in other words the origindestination of the goods passing through it.

- In accordance with its intermodality (monomodal or multimodal: road / rail / sea / air) depending on the modes of transport it brings together.

- In terms of its spatial concentration (concentrated or multicentre), namely whether it is located at one point or if it is dispersed over more than one space.

- And with respect to its range of activity (multifunctional or specialised), that is to say whether or not it depends on the demands of one sector.

- Likewise, for a logistics centre to be defined as such it must include at least the following functions or services:

- Logistics Function or Area: a load consolidation, splitting and storage centre; a logistical storage and distribution centre; an international transport centre and duty-free area (optional) and an intermodal centre (optional).

- Vehicle Servicing Function or Area: a parking area for trucks, a refuelling facility and repair workshops.

- Driver Care Function or Accommodation Area: a rest area, showers, bar-restaurant and hotel.

This means that the logistics centre is not defined according to its size, which is how the international community does it, but in accordance with concepts we consider to be more rational, namely the activity it performs and the way in which said activity is implemented.

With respect to the design parameters, it is necessary to establish the functional areas to be developed within the logistics centre and to standardise the percentages of each of these over the total surface.

In light of the results obtained, the authors conclude the following development percentages of each functional area and building coefficients:

- Logistic area: $40.00 \%$ of surface area over the total and building coefficient of $0.970 \mathrm{~m}^{2} / \mathrm{m}^{2}$

- Vehicle service area: $2.50 \%$ of surface area over the total and building coefficient of $1.099 \mathrm{~m}^{2} / \mathrm{m}^{2}$

- Service and administration Centre: $5.00 \%$ of surface area over the total and building coefficient of $1.075 \mathrm{~m}^{2} / \mathrm{m}^{2}$

- Truck Parking Space: $5.00 \%$ of surface area over the total

- Road Network: $19.50 \%$ of surface area over the total

- Green Areas: $18.00 \%$ of surface area over the total

- Other: $10.00 \%$ of surface area over the total and building coefficient of $0.414 \mathrm{~m}^{2} / \mathrm{m}^{2}$

Analysed and weighted the results obtained from all the logistic platforms studied, it is agreed that the optimal standards for the functional areas of a logistic platform type of 735,000 $\mathrm{m}^{2}$ of total surface are: 
- Standard conditions of plot and construction of the LOGISTIC AREA:

- Minimum surface: 4,200 $\mathrm{m}^{2}$

- Minimum plot's front: $28 \mathrm{~m}$

- Maximum capacity: $75 \%$

- Building coefficient: $0.970 \mathrm{~m}^{2} / \mathrm{m}^{2}$

- Total surface: $294,000 \mathrm{~m}^{2}$

- Buildable surface area: 285,180 $\mathrm{m}^{2}$

- Building height maximum $(\mathrm{H}): 21 \mathrm{~m}$

- Building's maximum number or floor: 4

Standard conditions of plot and construction of the VEHICLE SERVICE AREA:

- Minimum surface: 4,200 $\mathrm{m}^{2}$

- Minimum plot's front: $28 \mathrm{~m}$

- Maximum capacity: $65 \%$

- Building coefficient: $1.099 \mathrm{~m}^{2} / \mathrm{m}^{2}$

- Total surface: $18,375 \mathrm{~m}^{2}$

- Buildable surface area: 20,194 $\mathrm{m}^{2}$

- Building height maximum $(\mathrm{H}): 16 \mathrm{~m}$

- Building's maximum number or floor: 4

Standard conditions of plot and construction of the Service and Administration Centre:

Minimum surface: $3,300 \mathrm{~m}^{2}$

Minimum plot's front: $28 \mathrm{~m}$

Maximum capacity: $60 \%$

Building coefficient: $1.075 \mathrm{~m}^{2} / \mathrm{m}^{2}$

Total surface: $36,750 \mathrm{~m}^{2}$

Buildable surface area: 39,506 $\mathrm{m}^{2}$

Building height maximum $(\mathrm{H}): 15 \mathrm{~m}$

Building's maximum number or floor: 5

Finally, the road-rail intermodality must be strengthened as its presence is minimal in Spain's logistics platforms. This would contribute towards achieving the objectives set by the European Union with respect to optimising both the performance of the multimodal logistics chains and the creation of multimodal freight corridors for a sustainable transport network.

Asociación de Centros de Transporte de España. (n.d.). Retrieved from http://www.acte.es/catalogacion/.

Boletín Oficial del Estado. (2013). Ley 16/1987, de 30 de julio, de Ordenación de los Transportes Terrestre. Boletin Oficial del Estado, 160, 1-74.

Coca, P., Colomer, J., \& Aznar, J. (2010). El Transporte Terrestre de Pasajeros. Valencia, Spain. Fundacion Valenciaport.

Comisión Europea. (2001). La política europea de transportes de cara la 2010: la hora de la verdad. COM(2001) 370.

European Commission. (1992). El curso futuro de la política común de transportes. EC COM(92) 494.

Higgins, C. D., Ferguson, M., \& Kanaroglou, P. S. (2012). Varieties of Logistics Centers Developing Standardized Typology and Hierarchy. In Transportation Research Board of the National Academies (pp. 1-20).

Meidute, I. (2005). Comparative analysis of the definitions of logistics centres. Transport, 20(February 2015), $106-110$.

Ministerio de Fomento. (2005). Plan Estratégico de Infraestructuras y Transporte (PEIT). Madrid, Spain.

Ministerio de Fomento. (2012). Plan de infraestructuras, transporte y vivienda 2012 - 2024. Madrid, Spain.

Notteboom, T., \& Rodrigue, J. (2009). Inland terminals within North American and European supply chains. Transport and Communications Bulletin for Asia and the Pacific, 78(1), 1-39.

Ragàs Prat, I. (2012). Centros logísticos : planificación, promoción y gestión de los centros de actividades logísticas. (Margebooks, Ed.). Barcelona, Spain.

Rimienè, K., \& Grundey, D. (2007). Logistics Centre Concept through Evolution and Definition. Engineering Economics, 4(4), 87-95. 
Rodrigue, J., Debrie, J., \& Fremont, A. (2010). Functions and Actors of Inland Ports : European and North American Dynamics Functions and Actors of Inland Ports : European and North American Dynamics. Journal of Transport Geography, 18 (4), 519-529.

The European Logistics Platforms Association. (n.d.) Retrieved from http://www.europlatforms.eu/?page_id=150.

Tsamboulas, D. A., \& Dimitropoulos, I. (1999). Appraisal of investments in European nodal centres for goods - freight villages: A comparative analysis. Transportation, 26(4), 381-398.

Unión Europea (2011). Libro blanco del Transporte. Hoja de ruta hacia un espacio único europeo de transporte: por una política de transportes competitiva y sostenible. Luxembourg, Belgium. 